\title{
Magnetoroton Softening in Rb Spinor Condensates with Dipolar Interactions
}

\section{Citation}

Cherng, R. W., and E. Demler. 2009. "Magnetoroton Softening in Rb Spinor Condensates with Dipolar Interactions." Physical Review Letters 103 (18) (October 27). doi:10.1103/ physrevlett.103.185301.

\section{Published Version}

doi:10.1103/PhysRevLett.103.185301

\section{Permanent link}

http://nrs.harvard.edu/urn-3:HUL.InstRepos:26370383

\section{Terms of Use}

This article was downloaded from Harvard University's DASH repository, and is made available under the terms and conditions applicable to Other Posted Material, as set forth at http:// nrs.harvard.edu/urn-3:HUL.InstRepos:dash.current.terms-of-use\#LAA

\section{Share Your Story}

The Harvard community has made this article openly available.

Please share how this access benefits you. Submit a story.

\section{Accessibility}




\title{
Magnetoroton Softening in Rb Spinor Condensates with Dipolar Interactions
}

\author{
R. W. Cherng and E. Demler \\ Physics Department, Harvard University, Cambridge, Massachusetts 02138, USA \\ (Received 3 April 2009; revised manuscript received 9 October 2009; published 27 October 2009)

\begin{abstract}
Superfluids with a tendency towards periodic order have both phonon- and rotonlike spectra. We show that magnetoroton softening occurs in ${ }^{87} \mathrm{Rb}$ spinor condensates. A rich variety of dynamical instabilities emerges as a function of the magnetic field orientation and strength of the quadratic Zeeman shift. These instabilities are driven by an effective dipolar interaction modified dramatically by quasi-twodimensionality and rapid Larmor precession.
\end{abstract}

DOI: 10.1103/PhysRevLett.103.185301

PACS numbers: 67.85. $-\mathrm{d}, 03.75 . \mathrm{Kk}, 03.75 . \mathrm{Mn}$

Demonstrating the coexistence of long-range phase coherence and translational symmetry breaking in the exotic supersolid phase has been a long and elusive goal [1-6]. Theoretical interest dates back to early studies of superfluidity in ${ }^{4} \mathrm{He}$. Landau suggested that the excitation spectrum consists of two parts: a soundlike long-wavelength mode and a roton spectrum $\epsilon(p)=\Delta+\left(p-p_{0}\right)^{2} / 2 m$ for $p \approx p_{0}$ indicative of a tendency towards periodic order. The softening of the roton gap where $\Delta$ approaches zero provides one means of translational symmetry breaking without destroying phase coherence $[7,8]$.

In contrast to bulk ${ }^{4} \mathrm{He}$, current experiments $[9,10]$ on $F=1$ ferromagnetic condensates are in a quasi-twodimensional geometry. The spin healing length is larger than the Thomas-Fermi (TF) radius along the direction of tightest confinement while it is smaller than the TF radius along the two remaining directions. Spin degrees of freedom are two dimensional and undergo rapid Larmor precession due to an applied magnetic field. These effects strongly modify the effective dipolar interaction.

The role of dipolar interactions is well known in condensed matter systems [11], but spinor condensates offer additional competing and controllable interactions [12]. These include the quadratic Zeeman shift and spindependent contact interactions on the scale of a few $\mathrm{Hz}$. Previous studies have analyzed the tunability of dipolar interactions via time-dependent precession [13] but have not explored the implications for collective modes or dynamical instabilities. The role of the bare dipolar interactions for instabilities in quasi-two-dimensional single component quantum gases of polar molecules [14-18] or spinor condensates [19-21] has also been studied. In this Letter, we provide a unified treatment of dipolar interactions in quantum gases taking into account reduced dimensionality and rapid Larmor precession within a multicomponent description.

Our main results are summarized in Fig. 1. We consider a ferromagnet with an initially uniform magnetization $\vec{F}$. The collective mode spectrum has magnetorotonlike parts which can become imaginary at finite wave vectors indicating a dynamical instability. We trace the origin of these instabilities to two types of magnetization fluctuations: trans- verse fluctuations $\delta \vec{F}_{\perp}$ and longitudinal fluctuations $\delta \vec{F}_{\|}$. Here transverse (longitudinal) refers to spin components or wave vectors perpendicular (parallel) to the magnetic field along $\hat{B}$. For single component condensates, collapse dynamics driven by dipolar instabilities in single component condensates were directly observed in Ref. [22]. For multicomponent condensates, dipolar instabilities were analyzed within a long-wavelength effective theory [23].

Recall dipolar interactions favor spins aligned head to tail and antialigned side by side. Thus transverse fluctuations of magnetization, $\delta \vec{F}_{\perp}$, are energetically favorable for wave vectors parallel to the magnetic field and longitudinal fluctuations of magnetization, $\delta \vec{F}_{\|}$, are energetically favorable for wave vectors perpendicular to the
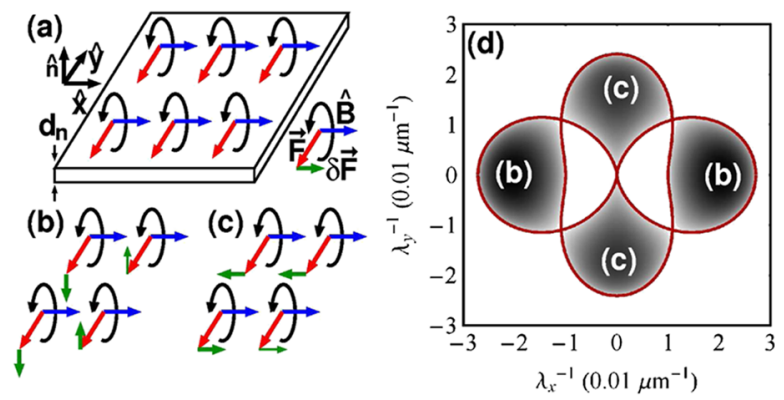

FIG. 1 (color online). (a) Quasi-two-dimensional spinor condensates with $\hat{x}, \hat{y}$ in plane confined to thickness $d_{n}$ along $\hat{n}$ out of plane. Magnetic field along $\hat{B}=\cos (\alpha) \hat{n}+\sin (\alpha) \hat{x}$ induces rapid Larmor precession (black circle) of a uniform magnetization $\vec{F}$ and quadratic Zeeman shift $q$. Precession and reduced dimensionality dramatically modify the effective dipolar interaction. Fluctuations $\delta \vec{F}$ can lower the classical dipolar energy and induce dynamical instabilities. $\hat{B}, \vec{F}$, and $\delta \vec{F}$ are depicted in the lower right corner. These occur for either (b) $\delta \vec{F}_{\perp}$ spin components perpendicular to $\hat{B}$ along $k_{\|}$wave vectors parallel to $\hat{B}$ or (c) $\delta \vec{F}_{\|}$spin components parallel to $\hat{B}$ along $k_{\perp}$ wave vectors perpendicular to $\hat{B}$. (d) Spin mode collective mode spectrum in momentum space for current experimental parameters $\alpha=\pi / 2, q=1.5 \mathrm{~Hz}$ [9]. Shading indicates the magnitude of $\operatorname{Im} \omega_{\vec{k}}$ for dynamically unstable modes. Regions labeled by corresponding unstable fluctuations. Notice the distinctive crosslike structure for unstable modes and stable region at the center. 
magnetic field (see Fig. 1). Individually, these two types of fluctuations can drive dynamical instabilities by lowering the effective classical dipolar energy. However, when both types are unstable at the classical level with negative energies, we show that the collective mode spectrum is actually stable. This is due to the classically conjugate nature of these fluctuations.

Including the effects of competing interactions allows us to construct a phase diagram as a function of quadratic Zeeman shift and magnetic field orientation. This phase diagram exhibits a variety of dynamical instabilities including those towards states with striped or checkerboard order. We plot a representative collective mode spectrum describing current experiments in Fig. 1.

Notice the distinctive crosslike structure strikingly similar to the observed magnetization correlations in $[9,10]$. In these experiments, the size of individual spin domains is $10 \mu \mathrm{m}$ while neighboring domains are modulated along an axis that is constant on a longer length scale of $20 \mu \mathrm{m}$. We find a characteristic length scale of 30-40 $\mu \mathrm{m}$ for the most unstable modes which compares favorably with the latter.

We consider a quasi-two-dimensional spinor condensate with unit vectors $\hat{x}, \hat{y}(\hat{n})$ in plane (out of plane), and magnetic field along $\hat{B}=\cos (\alpha) \hat{n}+\sin (\alpha) \hat{x}$. From here on, we refer to spin components parallel (perpendicular) to $\hat{B}$ as longitudinal (transverse). The in-plane component of $\hat{B}$ is along $\hat{x}$ so we also refer to real or momentum space components along $\hat{x}(\hat{y})$ as longitudinal (transverse). The Hamiltonian and Lagrangian are

$$
\begin{aligned}
\mathcal{H}= & \int d^{3} x \boldsymbol{\Psi}_{\vec{x}}^{\dagger}\left[-\frac{\nabla^{2}}{2 m}-\mu+B_{0} \hat{B} \cdot \vec{F}+q(\hat{B} \cdot \vec{F})^{2}\right] \boldsymbol{\Psi}_{\vec{x}} \\
& +\int d^{3} x\left[\frac{g_{0}}{2}: \boldsymbol{\Psi}_{\vec{x}}^{\dagger} \boldsymbol{\Psi}_{\vec{x}} \boldsymbol{\Psi}_{\vec{x}}^{\dagger} \boldsymbol{\Psi}_{\vec{x}}:+\frac{g_{s}}{2}: \boldsymbol{\Psi}_{\vec{x}}^{\dagger} \boldsymbol{\Psi}_{\vec{x}}^{*} \boldsymbol{\Psi}_{\vec{x}}^{T} \boldsymbol{\Psi}_{\vec{x}}:\right] \\
& +\int d^{3} x d^{3} x^{\prime} \frac{g_{d}}{2} h_{3 \mathrm{D}}^{i j}\left(\vec{x}-\vec{x}^{\prime}\right): \boldsymbol{\Psi}_{\vec{x}}^{\dagger} \vec{F}^{i} \boldsymbol{\Psi}_{\vec{x}} \boldsymbol{\Psi}_{\vec{x}^{\prime}}^{\dagger} \vec{F}^{j} \boldsymbol{\Psi}_{\vec{x}^{\prime}}: \\
\mathcal{L}= & \int d^{3} x\left[i \boldsymbol{\Psi}^{\dagger} \partial_{t} \boldsymbol{\Psi}\right]-\mathcal{H}
\end{aligned}
$$

where : : denotes normal ordering. Here $\boldsymbol{\Psi}_{\alpha}$ with $\alpha=1,2$, 3 are annihilation operators for $F=1$ bosons with mass $m$ and $\vec{F}$ hyperfine spin operators with $\vec{F}_{j k}^{i}=-i \epsilon_{i j k}$. Throughout, we use a matrix notation with suppressed indices where $*, T$, and $\dagger$ denote the complex conjugate, transpose, and the conjugate transpose, respectively. For example, $\boldsymbol{\Psi}\left(\boldsymbol{\Psi}^{\dagger}\right)$ is a column (row) vector while $\vec{F}^{i}$ is a matrix.

The chemical potential $\mu$ controls the density $\boldsymbol{\Psi}^{\dagger} \boldsymbol{\Psi}=$ $n_{3 \mathrm{D}}$ and we work with fixed longitudinal magnetization $\boldsymbol{\Psi}^{\dagger} \hat{B} \cdot \vec{F} \boldsymbol{\Psi}=n_{3 \mathrm{D}} f_{B}$. The magnetic field induces rapid Larmor precession about $\hat{B}$ at a frequency $B_{0}$ and a quadratic Zeeman shift $q$. With typical $B$ of zero up to hundreds of $\mathrm{mG}, q=70 \mathrm{~Hz} \mathrm{G}^{-2} B^{2}$ [12] ranges from zero to tens of Hz. ac Stark shifts due to properly detuned circularly polarized can further tune $q$ by acting as an effective Zeeman shift [24]. A harmonic trapping potential along $\hat{n}$ confines the condensate to a thickness $d_{n}$. We take typical values of $B_{0} / 2 \pi=115 \mathrm{kHz}$ and $d_{n}=2 \mu \mathrm{m}$ [9].
The spin-independent and spin-dependent contact interaction strengths are given by $g_{0}=4 \pi \hbar^{2} a_{0} / m, g_{s}=$ $4 \pi \hbar^{2}\left(a_{0}-a_{2}\right) / 3 m$ [25] in terms of the $s$-wave scattering lengths $a_{F}$ for two atoms colliding with total angular momentum $F$. For ${ }^{87} \mathrm{Rb}, a_{0}=101.8 a_{B}$ and $a_{2}=$ $100.4 a_{B}$ where $a_{B}$ is the Bohr radius [26] giving positive $g_{s}$ and ferromagnetic interactions. The dipolar interaction strength is given by $g_{d}=\mu_{0} g_{F}^{2} \mu_{B}^{2} / 4 \pi$ where $\mu_{0}$ is the vacuum permeability, $g_{F}$ is the Landé $g$ factor, and $\mu_{B}$ is the Bohr magneton while the dipolar interaction tensor is given by

$$
h_{3 \mathrm{D}}^{i j}(\vec{x})=\frac{1}{x^{3}} P^{i j}(\hat{x}), \quad h_{3 \mathrm{D}}^{i j}(\vec{k})=-\frac{4 \pi}{3} P^{i j}(\hat{k})
$$

in real and momentum space with $P^{i j}(\hat{z})=\delta^{i j}-3 \hat{z}^{i} \hat{z}^{j}$. For typical peak three-dimensional densities of $n_{3 \mathrm{D}}=2.2 \times$ $10^{14} \mathrm{~cm}^{-3}$ the interaction strengths are $g_{0} n_{3 \mathrm{D}}=1.7 \mathrm{kHz}$, $g_{s} n_{3 \mathrm{D}}=8 \mathrm{~Hz}$, and $g_{d} n_{3 \mathrm{D}}=0.8 \mathrm{~Hz}[9,12]$.

The quadratic Zeeman shift, spin-dependent contact interactions, and dipolar interactions govern the lowenergy in-plane spin degrees of freedom at a scale of a few Hz. This energy scale is well separated from that of spin-independent contact interactions, rapid Larmor precession, and out-of-plane confinement. The effect of these terms on the low-energy degrees of freedom is to fix the local density, induce spatial averaging on a scale $d_{n}$ along $\hat{n}$, and induce time averaging on a scale $B_{0}^{-1}$, respectively.

We thus assume a condensate frozen along $\hat{n}$ with fixed local density and transform to a rotating frame comoving with respect to Larmor precession

$$
\boldsymbol{\Psi}_{\vec{x}} \rightarrow \sqrt{n_{3 \mathrm{D}} d_{n} \rho\left(x_{n} / d_{n}\right)} R\left(B_{0} t\right) \boldsymbol{\Psi}_{\vec{x}},
$$

where $\boldsymbol{\Psi}_{\vec{x}}$ on the left (right) is a three-dimensional (twodimensional) field in the lab (comoving) frame with $x_{n}$ along $\hat{n}$ and $R(\tau)=\exp (-i \tau \hat{B} \cdot \vec{F})$. For definiteness we consider a Gaussian profile $\rho(z)=\exp \left(-z^{2} / 2\right) / \sqrt{2 \pi}$.

Substituting Eq. (3) into the Lagrangian of Eq. (1) and integrating over $x_{n}$ and one period $\Delta t=2 \pi / B_{0}$ yields $d^{3} x \rightarrow d^{2} x, B_{0} \rightarrow 0, g_{i} \rightarrow g_{i} n_{3 \mathrm{D}} d_{n} \rho(0) C$ where $i=0, s$, $d$, the constant $C=1 / \sqrt{2}$ is determined by normalization, and $\boldsymbol{\Psi}$ satisfies $\boldsymbol{\Psi}^{\dagger} \boldsymbol{\Psi}=1, \boldsymbol{\Psi}^{\dagger} \hat{B} \cdot \vec{F} \boldsymbol{\Psi}=f_{B}$. The effective dipolar interaction tensor in momentum space $h_{3 \mathrm{D}}^{i j}(\vec{k}) \rightarrow h_{2 \mathrm{D}}^{i j}(\vec{k})$ is given by

$$
\begin{aligned}
h_{2 \mathrm{D}}^{i j}(\vec{k}) & =\left\langle e^{i k_{n} d_{n}\left(z-z^{\prime}\right)} R_{i l}^{T}(\tau) h_{3 \mathrm{D}}^{l m}\left(\vec{k}+k_{n} \hat{n}\right) R_{m j}(\tau)\right\rangle, \\
\langle f\rangle & =\frac{\int_{-\pi}^{+\pi} d \tau \int d z d z^{\prime} \rho(z) \rho\left(z^{\prime}\right) f\left(\tau, z, z^{\prime}\right)}{\int_{-\pi}^{+\pi} d \tau \int d z d z^{\prime} \rho(z) \rho\left(z^{\prime}\right)}
\end{aligned}
$$

and the averaging can be performed explicitly yielding

$$
\begin{aligned}
h_{2 \mathrm{D}}^{i j}(\vec{k}) & =-\frac{4 \pi}{3}\left[\frac{3 h\left(\vec{k} d_{n}\right)-1}{2}\right]\left[\delta^{i j}-3 \hat{B}^{i} \hat{B}^{j}\right], \\
h(\vec{k}) & =[\hat{B} \cdot \hat{k}]^{2} w(k)+[\hat{B} \cdot \hat{n}]^{2}[1-w(k)], \\
w(x) & =2 x \int_{0}^{\infty} d z e^{-\left(z^{2}+2 z x\right)}
\end{aligned}
$$

with $w(x)$ rather insensitive to details of $\rho(z)$. 
This effective dipolar interaction can drive dynamical instabilities in a uniform ferromagnet. Bogoliubov analysis of the collective mode spectrum provides a systematic approach to studying such instabilities. We take $\hat{B}$ as the quantization axis and parametrize

$$
\boldsymbol{\Psi}_{\vec{x}}=\sqrt{n_{\vec{x}}}\left[\begin{array}{c}
i e^{i \eta_{\vec{x}}+i \nu_{\vec{x}}} \cos \left(\phi_{\vec{x}}+i \chi_{\vec{x}}\right) \frac{\sin \left(\rho_{\vec{x}}\right)}{\sqrt{\cosh \left(2 \chi_{\vec{x}}\right)}} \\
i e^{i \eta_{\vec{x}}+i \nu_{\vec{x}}} \sin \left(\phi_{\vec{x}}+i \chi_{\vec{x}}\right) \frac{\sin \left(\rho_{\vec{x}}\right)}{\sqrt{\cosh \left(2 \chi_{\vec{x}}\right)}} \\
e^{i \eta_{\vec{x}}} \cos \left(\rho_{\vec{x}}\right)
\end{array}\right]
$$

with $n$ the two-dimensional density, $\eta$ the global phase, $\rho$, $\nu$ controlling the magnitude of the magnetization, $\phi$ the orientation of the transverse magnetization, and $\chi$ the magnitude of the longitudinal magnetization. For $\boldsymbol{\Psi}_{\vec{x}}=$ $\boldsymbol{\Psi}$ independent of $\vec{x}$ we find for ferromagnetic interactions $g_{s}>0$ the mean-field energy is independent of $\eta$ and $\phi$ and minimized at $\nu=0$. Introducing the quantities

$$
g_{\perp}(\vec{k})=g_{s}-\frac{2 \pi}{3} g_{d}\left[3 h\left(\vec{k} d_{n}\right)-1\right]
$$

and $Q=q / 2 g_{\perp}(0) n_{3 \mathrm{D}} C$ we find the polar state for $|Q|>1$ and ferromagnet for $|Q| \leq 1$.

To study collective modes, we focus on the $f_{B}=0$ case relevant for current experiments. Taking $\boldsymbol{\Psi}_{\vec{x}}=\boldsymbol{\Psi}+\delta \boldsymbol{\Psi}_{\vec{x}}$ we use the Lagrangian of Eq. (1) to find the linearized equations of motion for small fluctuations $\delta \boldsymbol{\Psi}_{\vec{k}}$. With the parametrization of Eq. (6), $\delta n, \delta \eta, \delta \rho$, and $\delta \nu$ couple to form two branches of the collective mode spectrum. At small $k$, they describe a linearly dispersing Goldstone mode describing superfluid phonon excitations and a gapped mode describing fluctuations in the magnetization magnitude. These modes develop dynamical instabilities only very close to the mean-field transition between polar and ferromagnet states.

We instead focus on $\delta \phi, \delta \chi$ which decouple completely from $\delta n, \delta \eta, \delta \rho, \delta \nu$ and form a branch corresponding to the spin mode. The momentum space Lagrangian

$$
\begin{aligned}
\mathcal{L}= & i \delta \phi_{\vec{k}}^{*} \partial_{t} \delta \chi_{\vec{k}}-\left[E_{\phi}(\vec{k})\left|\delta \phi_{\vec{k}}\right|^{2}+E_{\chi}(\vec{k})\left|\delta \chi_{\vec{k}}\right|^{2}\right], \\
E_{\phi}(\vec{k})= & \frac{k^{2}}{2 m}-\left[(1+Q) g_{\perp}(\vec{k})-(1+Q) g_{\perp}(0)\right] n_{3 \mathrm{D}} C, \\
E_{\chi}(\vec{k})= & {\left[2(1-Q) g_{\perp}(\vec{k})+(1+Q) g_{\perp}(0)\right] n_{3 \mathrm{D}} C } \\
& +\frac{k^{2}}{2 m}+3(Q-1) g_{s} n_{3 \mathrm{D}} C
\end{aligned}
$$

describes these quadratic fluctuations.

Recall the transverse orientation $(\delta \phi)$ and longitudinal magnitude $(\delta \chi)$ of the magnetization are canonically conjugate. This is evident from the coupling $\delta \phi_{\vec{k}}^{*} \partial_{t} \delta \chi_{\vec{k}}$ of the above the Lagrangian which arise from the $i \boldsymbol{\Psi} \partial_{t} \boldsymbol{\Psi}$ term of the underlying time-dependent Gross-Pitaevskii equation. At the level of the classical energy in brackets, the fluctuations decouple into independent contributions $E_{\phi}(\vec{k})$ and $E_{\chi}(\vec{k})$. Explicitly, $\omega_{\vec{k}}^{2}=E_{\phi}(\vec{k}) E_{\chi}(\vec{k})$ is easily derived in terms of the classical energies. Thus we see that only when one but not both of the contributions $E_{\phi}(\vec{k}), E_{\chi}(\vec{k})$ is negative does $\omega_{\vec{k}}$ become imaginary giving a true dynamical instability.

We plot representative $\omega_{\vec{k}}$ in Fig. 2 illustrating the spin mode for $\alpha=\pi / 4$ near $q_{c}=-1.35 \mathrm{~Hz}$. Notice the appearance of a magnetoroton minimum and the softening of the magnetoroton gap as $q$ approaches $q_{c}$. When $q$ is below $q_{c}$, the spin mode becomes imaginary at finite wave vector. Note negative $q$ can be achieved via ac Stark shifts.

The imaginary part of the collective mode spectrum using current experimental parameters $\alpha=\pi / 2, q=$ $1.5 \mathrm{~Hz}$ is shown in Fig. 1. These parameters are chosen to best illustrate softening of the magnetoroton mode and are near the border between a dynamically stable and unstable uniform ferromagnet. Notice the similarity with the clear crosslike structure observed for the magnetization structure factor [9]. The dynamical instability for the transverse (longitudinal) spin component along longitudinal (transverse) momenta arises directly from the regions where the classical energy for transverse (longitudinal) spin fluctuations $E_{\phi}(\vec{k})\left(E_{\chi}(\vec{k})\right)$ is negative. When both are negative at small $k$, the spin mode is in fact stable reflecting the conjugate nature of these variables.

From our analysis, the length scale of the most unstable mode shown in Fig. 1 is $30-40 \mu \mathrm{m}$. Its physical origin is simply the competition between the $|k|$ scaling of gain in dipolar interaction energy versus $|k|^{2}$ scaling of cost in kinetic energy. The length scale at which these two contributions are comparable is given by

$$
l^{-1} \sim \frac{4 \pi}{3} g_{d} n_{3 \mathrm{D}} \rho(0) d_{n} m
$$

with $l \sim 30 \mu \mathrm{m}$.

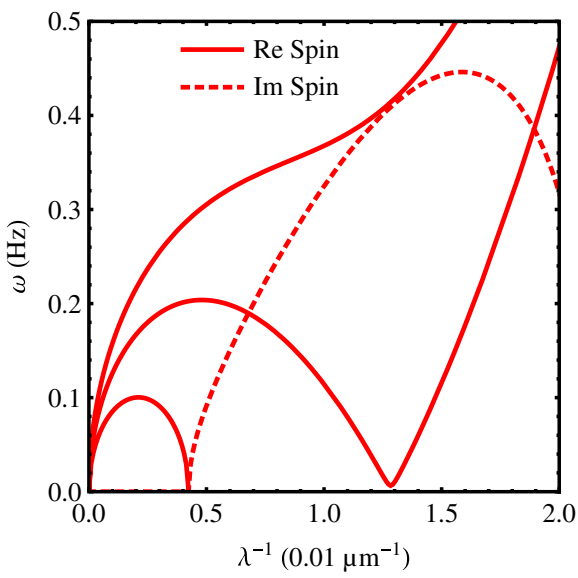

FIG. 2 (color online). Spin mode collective mode spectrum $\omega_{\vec{k}}$ along $k_{y}$ for magnetic field orientations $\alpha$ and quadratic Zeeman shifts $q(\mathrm{~Hz})(q, \alpha)=(-1.1,0.247 \pi),(-1.35,0.25 \pi),(-1.6$, $0.253 \pi)$ going from top to bottom. These parameters are near the border between a dynamically stable and unstable uniform ferromagnet and best illustrate softening of the roton minimum. Note negative $q$ can be obtained through ac Stark shifts [24]. 


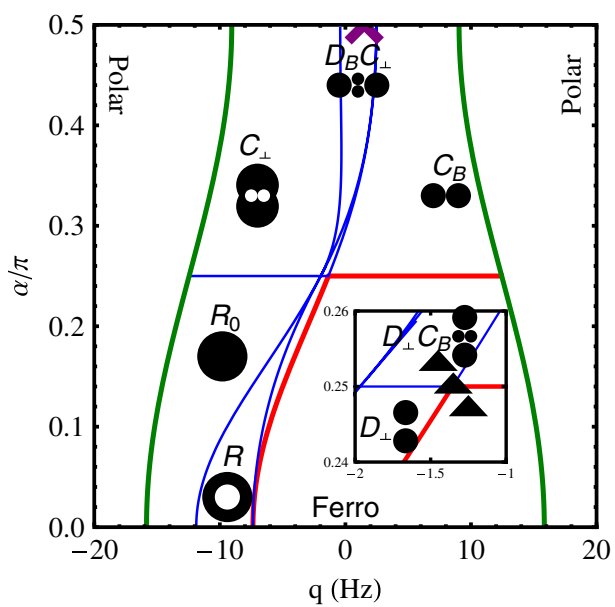

FIG. 3 (color online). Spin mode phase diagram as a function of magnetic field orientation $\alpha$ and quadratic Zeeman shift $q$ based on analysis of the collective mode spectrum. The uniform ferromagnet (polar state) is stable within (outside) the thick lines near the left and right edges at the mean-field level. The ferromagnet is dynamically stable (unstable) below (above) the thick line in the interior. The unstable modes occur in a variety of topologies in momentum space and regions with different topologies separated by thin lines. Illustrative plots of unstable modes in momentum space for each distinct topology (black circles) show the evolution from crosslike four-lobed structures $\left(D_{B} C_{\perp}, D_{\perp} C_{B}\right)$ to dumbbell-shaped two-lobed structures $\left(C_{B}\right.$, $\left.C_{\perp}, D_{\perp}\right)$ to ring structures $\left(R_{0}, R\right)$. Four-lobed (two-lobed) structures for unstable modes can seed the growth of checkerboard (striped) spin textures. The inset is of the region near $q=$ 1.5, $\alpha=\pi / 4$. The $X$ near the top center denotes parameters for current experiments. The black triangles are for parameters of Fig. 2.

Current experiments observe magnetization modulations with two characteristic length scales. Individual spin domains are typically $10 \mu \mathrm{m}$ in size. Neighboring domains appear to be modulated antiferromagnetically along a preferred spin axis. This spin axis is uniform on a larger length scale of $30 \mu \mathrm{m}$ [10]. One means of understanding the emergence of two length scales is as follows. Dynamical instabilities of the uniform state seed fluctuations that set the characteristic length scale of the unit cell in the final spin texture. However, higher order effects can stabilize smaller scale spin domains within each unit cell.

A similar analysis of the collective mode spectrum gives the phase diagram in Fig. 3 as a function of the quadratic Zeeman shift $q$ and orientation of the magnetic field $\alpha$ where $\alpha=0(\alpha=\pi / 2)$ corresponds to $\hat{B}$ out of the plane (in the plane). $R$ indicates a ring-shaped region of unstable modes, $R_{0}$ disk-shaped, $C_{\perp}\left(C_{B}\right)$ two connected regions parallel (perpendicular) to $\hat{B}, D_{\perp}\left(D_{B}\right)$ two disconnected regions perpendicular (parallel) to $\hat{B}, D_{\perp} C_{B}\left(D_{B} C_{\perp}\right)$ two disconnected regions parallel (perpendicular) to $\hat{B}$ along with two connected regions perpendicular (parallel) to $\hat{B}$.
In conclusion, we have shown the collective mode spectrum for fluctuations above a uniform ferromagnet shows both a phonon and magnetorotonlike spectrum as well as a variety of dynamical instabilities. The resulting phase diagram as a function of quadratic Zeeman shift and orientation of the magnetic field shows a rich variety of finite wave vector instabilities. The origin of these dynamical instabilities can be traced to canonically conjugate fluctuations in the transverse orientation and longitudinal magnitude of the magnetization. These fluctuations can lower the classical energy for the effective dipolar interaction. We also demonstrated how rapid Larmor precession and reduced dimensionality qualitatively alters this dipolar interaction.

We acknowledge useful discussions with D. StamperKurn, M. Vengalatorre, A. Lamacraft, V. Gritsev, G. Shlyapnikov, S. Girvin. This work was supported by NDSEG and the NSF Graduate Research program, Harvard-MIT CUA, DARPA, MURI, and NSF Grant No. DMR-0705472.

[1] A. F. Andreev and I. M. Lifshitz, Sov. Phys. JETP 29, 1107 (1969).

[2] G. V. Chester, Phys. Rev. A 2, 256 (1970).

[3] A. J. Leggett, Phys. Rev. Lett. 25, 1543 (1970).

[4] A. van Otterlo et al., Phys. Rev. B 52, 16176 (1995).

[5] P. Sengupta et al., Phys. Rev. Lett. 94, 207202 (2005).

[6] V. W. Scarola and S. D. Sarma, Phys. Rev. Lett. 95, 033003 (2005).

[7] D. Kirzhnits and Y. Nepomnyashchii, Sov. Phys. JETP 59, 2203 (1970).

[8] T. Schneider and C.P. Enz, Phys. Rev. Lett. 27, 1186 (1971).

[9] M. Vengalattore et al., Phys. Rev. Lett. 100, 170403 (2008).

[10] M. Vengalattore, J. Guzman, S. Leslie, F. Serwane, and D. M. Stamper-Kurn, arXiv:0901.3800.

[11] C. Kittel and E. Abrahams, Rev. Mod. Phys. 25, 233 (1953).

[12] L. E. Sadler et al., Nature (London) 443, 312 (2006).

[13] S. Giovanazzi et al., Phys. Rev. Lett. 89, 130401 (2002).

[14] L. Santos et al., Phys. Rev. Lett. 85, 1791 (2000).

[15] K. Goral and L. Santos, Phys. Rev. A 66, 023613 (2002).

[16] L. Santos et al., Phys. Rev. Lett. 90, 250403 (2003).

[17] U. Fischer, Phys. Rev. A 73, 031602 (2006).

[18] D.-W. Wang, New J. Phys. 10, 053005 (2008).

[19] H. Pu et al., Phys. Rev. Lett. 87, 140405 (2001).

[20] S. Yi and H. Pu, Phys. Rev. Lett. 97, 020401 (2006).

[21] Y. Kawaguchi et al., Phys. Rev. Lett. 97, 130404 (2006).

[22] T. Lahaye et al., Phys. Rev. Lett. 101, 080401 (2008).

[23] A. Lamacraft, Phys. Rev. A 77, 063622 (2008).

[24] C. Y. Park et al., Phys. Rev. A 63, 032512 (2001).

[25] T.-L. Ho, Phys. Rev. Lett. 81, 742 (1998).

[26] E. G. M. van Kempen et al., Phys. Rev. Lett. 88, 093201 (2002). 